\title{
Multiplicatively dependent vectors with coordinates algebraic numbers
}

\author{
C. L. Stewart
}

In memory of Professor Alan Baker

\begin{abstract}
We shall prove that close to each point in $\mathbb{C}^{n}$ with coordinates of comparable size there is a point $\left(t_{1}, \ldots, t_{n}\right)$ with the property that no multiplicatively dependent vector $\left(u_{1}, \ldots, u_{n}\right)$ with coordinates which are algebraic numbers of height at most $H$ and degree at most $d$ is very close to $\left(t_{1}, \ldots, t_{n}\right)$.
\end{abstract}

Keywords. Multiplicatively dependent vectors, heights

2010 Mathematics Subject Classification. 11N25, 11R04

\section{Introduction}

Let $n$ be an integer with $n \geq 2$ and $R$ be a ring with identity. A vector $\mathbf{v}=\left(v_{1} \ldots, v_{n}\right)$ in $R^{n}$ is said to be multiplicatively dependent if all its coordinates are non-zero and there is a non-zero integer vector $\mathbf{k}=\left(k_{1}, \ldots, k_{n}\right)$ for which

$$
\mathbf{v}^{\mathbf{k}}=v_{1}^{k_{1}} \cdots v_{n}^{k_{n}}=1 .
$$

Let $S$ be a subset of $R$. We denote by $\mathcal{M}_{n}(S)$ the set of multiplicatively dependent vectors with coordinates in $S$.

In [PSSS18] Pappalardi, Sha, Shparlinski and Stewart give asymptotic formulae for the number of multiplicatively dependent vectors of algebraic numbers of fixed degree, or within a fixed number field, and bounded height. For any algebraic number $\alpha$, let

$$
f(x)=a_{d} x^{d}+\cdots+a_{1} x+a_{0}
$$

be the minimal polynomial of $\alpha$, so with content 1 and positive leading coefficient. Suppose that $f$ factors as

$$
f(x)=a_{d}\left(x-\alpha_{1}\right) \cdots\left(x-\alpha_{d}\right)
$$

over the complex numbers. The height $H(\alpha)$ of $\alpha$ is given by

$$
H(\alpha)=\left(a_{d} \prod_{i=1}^{d} \max \left(1,\left|\alpha_{i}\right|\right)\right)^{1 / d}
$$

For positive integers $n, d$ and $H$ with $n \geq 2$ we denote by $M_{n, d}^{*}(H)$ the number of multiplicatively dependent $n$-tuples whose coordinates are algebraic numbers of degree $d$ and height at most $H$. Pappalardi, Sha, Shparlinski and Stewart proved that

$$
M_{n, d}^{*}(H)=C(n, d) H^{d(d+1)(n-1)}+O\left(H^{d(d+1)(n-1)-d / 2} \log H\right)
$$

This research was supported in part by Grant A3528 from the Natural Sciences and Engineering Research Council of Canada and by the Canada Research Chairs Program.

We thank episciences.org for providing open access hosting of the electronic journal Hardy-Ramanujan Journal 
where

$$
C(n, d)=\left(n w_{0}(d)+2 n(n-1)\right) C_{1}(d)^{n-1},
$$

$w_{0}(d)$ is the number of roots of unity of degree $d$,

$$
C_{1}(d)=\frac{d 2^{d}}{\zeta(d+1)} \prod_{j=1}^{\lfloor(d-1) / 2\rfloor} \frac{(d+1)(2 j)^{d-2 j}}{(2 j+1)^{d-2 j+1}}
$$

and $\zeta(s)$ is the Riemann zeta function.

Sha, Shparlinski and Stewart [SSS] studied the distribution of elements of $\mathcal{M}_{n}(S)$ in $\mathbb{R}^{n}$ and in $\mathbb{C}^{n}$ when $S$ is a number field or the ring of algebraic integers of a number field. They showed that $\mathcal{M}_{n}(\mathbb{Q})$ is dense in $\mathbb{R}^{n}$ and if $K$ is a number field which is not contained in $\mathbb{R}$ then $\mathcal{M}_{n}(K)$ is dense in $\mathbb{C}^{n}$. Further if $K$ is contained in $\mathbb{R}$ and of degree at least 2 over the rationals then $\mathcal{M}_{n}\left(\mathcal{O}_{K}\right)$ is dense in $\mathbb{R}^{n}$ where $\mathcal{O}_{K}$ denotes the ring of algebraic integers of $K$. Furthermore if $K$ is contained in $\mathbb{C}$ but not in $\mathbb{R}$ and of degree at least 3 over the rationals then $\mathcal{M}_{n}\left(\mathcal{O}_{K}\right)$ is dense in $\mathbb{C}^{n}$. In addition they showed that there are significant irregularities in the distribution of the points of $\mathcal{M}_{n}(\mathbb{Z})$ in $\mathbb{R}^{n}$ and the distribution of the points of $\mathcal{M}_{n}\left(\mathcal{O}_{K}\right)$ in $\mathbb{C}^{n}$ when $K$ is an imaginary quadratic field. For $\mathbf{z}=\left(z_{1}, \ldots, z_{n}\right)$ in $\mathbb{C}^{n}$ put

$$
\|\mathbf{z}\|=\sqrt{\left|z_{1}\right|^{2}+\cdots+\left|z_{n}\right|^{2}}
$$

and for a real number $H$ greater than 1 put

$$
\mu_{n}\left(H ; \mathcal{O}_{K}\right)=\sup _{\substack{\mathbf{x} \in \mathbb{C}^{n} \\\|\mathbf{x}\| \leq H}} \inf _{\mathbf{v} \in \mathcal{M}_{n}\left(\mathcal{O}_{K}\right)}\|\mathbf{x}-\mathbf{v}\|
$$

when $K$ is an imaginary quadratic field. Sha, Shparlinski and Stewart proved there is a positive number $C_{0}(n)$, which is effectively computable in terms of $n$, such that for $n \geq 3$

$$
\mu_{n}\left(H, \mathcal{O}_{K}\right) \gg H /(\log H)^{C_{0}(n)}
$$

and

$$
\mu_{2}\left(H, \mathcal{O}_{K}\right) \gg H .
$$

Furthermore, for $n \geq 4$, there exists a positive number $C_{1}(K)$, which is effectively computable in terms of $K$, such that

$$
\mu_{n}\left(H ; \mathcal{O}_{K}\right) \ll H /(\log H)^{C_{1}(K)} .
$$

The purpose of this note is to show, extending estimate (1.3), that the multiplicatively dependent vectors in $\mathbb{C}^{n}$ with coordinates which are algebraic numbers of degree at most $d$ and height at most $H$ are distributed in a very irregular manner. We shall prove that throughout $\mathbb{C}^{n}$ there are many large polydiscs which have no elements of $\mathcal{M}_{n}(\mathbb{C})$ whose coordinates are algebraic numbers of degree at most $d$ and height at most $H$. In particular we shall show that close to every point of $\mathbb{C}^{n}$, with $n \geq 2$, with coordinates which are not too small there is a point which does not have any very close points from $\mathcal{M}_{n}(\mathbb{C})$ whose coordinates are algebraic numbers of degree at most $d$ and height at most $H$. We shall prove the following result.

Theorem 1.1. Let $n$ and $d$ be positive integers with $n \geq 2$. Let $\varepsilon$ and $H$ be real numbers with $0<\varepsilon<1$ and $H \geq 3$. There exist positive numbers $c, c_{0}$ and $C$, which are effectively computable in terms of $n$, of $n$ and $\varepsilon$, and of $n, d$ and $\varepsilon$ respectively, such that if $H$ exceeds $C$ and $\mathbf{z}=\left(z_{1}, \ldots, z_{n}\right)$ in $\mathbb{C}^{n}$ satisfies

$$
e^{(\log H)^{\varepsilon}}<\left|z_{j}\right| \leq H
$$

for $j=1, \ldots, n$ then there exists $\mathbf{t}=\left(t_{1}, \ldots, t_{n}\right)$ in $\mathbb{C}^{n}$ with

$$
\left|z_{j}-t_{j}\right|<\left|z_{j}\right| /\left(\log \left|z_{j}\right|\right)^{c}
$$


for $j=1, \ldots, n$ and such that there is no element $\mathbf{u}=\left(u_{1}, \ldots, u_{n}\right)$ in $\mathcal{M}_{n}(\mathbb{C})$ with coordinates which are algebraic numbers of height at most $H$ and degree at most $d$ for which

$$
\left|u_{j}-t_{j}\right|<\left|t_{j}\right| /\left(\log \left|t_{j}\right|\right)^{c_{0}}
$$

for $j=1, \ldots, n$.

For the proof of Theorem 1.1 we shall require a result of Loxton and van der Poorten [LoxP83, PLox77] which states that if $\alpha_{1}, \ldots, \alpha_{n}$ are algebraic numbers which are multiplicatively dependent then we can find a dependence relation where the exponents are not too large. In addition we need two results of Stewart [Ste18] which generalize earlier work of Tijdeman [Tij73, Tij74] on the sequence of integers generated by a finite set of primes. The results [Ste18, Tij73, Tij74] depend on the fundamental work of Baker on estimates for linear forms in the logarithms of algebraic numbers.

\section{Preliminary lemmas}

We first state the result of Loxton and van der Poorten [LoxP83, PLox77].

Lemma 2.1. Let $n \geq 2$ and let $\alpha_{1}, \ldots, \alpha_{n}$ be multiplicatively dependent non-zero algebraic numbers of degree at most $d$ which are not roots of unity. Then there is a positive number $c$, which depends only on $n$ and $d$, and there are rational integers $k_{1}, \ldots, k_{n}$, not all zero, such that

$$
\alpha_{1}^{k_{1}} \cdots \alpha_{n}^{k_{n}}=1
$$

and

$$
\left|k_{j}\right| \leq c \prod_{m=1, m \neq j}^{n} \log H\left(\alpha_{j}\right), \quad j=1, \ldots, n .
$$

Next we shall need Theorem 1 of [Ste18].

Lemma 2.2. Let $\alpha_{1}, \ldots, \alpha_{r}$ be multiplicatively independent algebraic numbers with $\left|\alpha_{i}\right|>1$ for $i=$ $1, \ldots, r$. Put

$$
T=\left\{\alpha_{1}^{h_{1}} \cdots \alpha_{r}^{h_{r}} \mid h_{i} \geq 0 \text { for } i=1, \ldots, r\right\} .
$$

There exists a positive number $c$, which is effectively computable in terms of $\alpha_{1}, \ldots, \alpha_{r}$, such that if $t$ and $t^{\prime}$ are in $T$ with $|t| \geq 3$ then

$$
\left|t-t^{\prime}\right|>|t| /(\log |t|)^{c} .
$$

We shall also require Theorem 3 of [Ste18].

Lemma 2.3. Let $\alpha_{1}, \alpha_{2}$ and $\alpha_{3}$ be multiplicatively independent algebraic numbers with $\left|\alpha_{i}\right|>1$ for $i=1,2,3$. Suppose that $\alpha_{1}$ and $\alpha_{2}$ are positive real numbers and that $\alpha_{3} /\left|\alpha_{3}\right|$ is not a root of unity. Put

$$
T=\left\{\alpha_{1}^{h_{1}} \alpha_{2}^{h_{2}} \alpha_{3}^{h_{3}} \mid h_{i} \geq 0 \text { for } i=1,2,3\right\} .
$$

There exists a positive number $c_{1}$, which is effectively computable in terms of $\alpha_{1}, \alpha_{2}$ and $\alpha_{3}$, such that for any complex number $z$ with $|z| \geq 3$ there exists an element $t$ of $T$ with

$$
|z-t| \leq|z| /(\log |z|)^{c_{1}} .
$$




\section{Proof of Theorem 1.1}

Let $r_{1}, r_{2}, \ldots$, be the increasing sequence of primes congruent to 1 modulo 4 . Let

$$
r_{j}=a_{j}^{2}+b_{j}^{2}
$$

with $a_{j}$ and $b_{j}$ positive integers for $j=1,2, \ldots$ and put

$$
\pi_{j}=a_{j}+i b_{j}
$$

for $j=1,2, \ldots$ Notice that

$$
\left|\pi_{j}\right|>1
$$

and that $\pi_{j} /\left|\pi_{j}\right|$ is not a root of unity since the only rational prime which ramifies in $\mathbb{Q}(i)$ is 2 . Let $q_{1}, q_{2}, \ldots$ be the increasing sequence of primes congruent to 3 modulo 4 . Let $H$ and $\varepsilon$ be real numbers with $0<\varepsilon<1$ and $H \geq 3$ and suppose that $\mathbf{z}=\left(z_{1}, \ldots, z_{n}\right)$ in $\mathbb{C}^{n}$ satisfies (1.4). Assume that $C$ is sufficiently large that $(\log C)^{\varepsilon} \geq \log 3$ and that $H$ exceeds $C$. Then, by Lemma 2.3, for each integer $j$ with $1 \leq j \leq n$ there exists a positive number $c_{j}$, which is effectively computable in terms of $q_{2 j-1}$, $q_{2 j}$ and $\pi_{j}$, and non-negative integers $\lambda_{j, 1}, \lambda_{j, 2}, \lambda_{j, 3}$ such that

$$
\left|z_{j}-q_{2 j-1}^{\lambda_{j, 1}} q_{2 j}^{\lambda_{j, 2}} \pi_{j}^{\lambda_{j, 3}}\right|<\frac{\left|z_{j}\right|}{\left(\log \left|z_{j}\right|\right)^{c_{j}}} .
$$

Put

$$
t_{j}=q_{2 j-1}^{\lambda_{j, 1}} q_{2 j}^{\lambda_{j, 2}} \pi_{j}^{\lambda_{j, 3}}
$$

for $j=1, \ldots, n$ and

$$
\mathbf{t}=\left(t_{1}, \ldots, t_{n}\right)
$$

Then, by (3.8),

$$
\left|z_{j}-t_{j}\right|<\left|z_{j}\right| /\left(\log \left|z_{j}\right|\right)^{c}
$$

where

$$
c=\min _{1 \leq j \leq n} c_{j}
$$

Observe that $c$ is effectively computable in terms of $n$ and so (1.5) holds.

Let $\mathbf{u}=\left(u_{1}, \ldots, u_{n}\right)$ be an element of $\mathcal{M}_{n}(\mathbb{C})$ with coordinates algebraic numbers of height at most $H$ and degree at most $d$. We shall prove that $\mathbf{u}$ is not close to $\mathbf{t}$. Further we note that $\mathbf{t}$ is not in $\mathcal{M}_{n}(\mathbb{C})$.

Let $C_{1}, C_{2}, \ldots$ be positive numbers which are effectively computable in terms of $n, d$ and $\varepsilon$. By Lemma 2.1 there exist integers $k_{1}, \ldots, k_{n}$, not all 0 , with

$$
\left|k_{j}\right| \leq C_{1}(\log H)^{n-1}
$$

for $j=1, \ldots, n$ for which

$$
u_{1}^{k_{1}} \cdots u_{n}^{k_{n}}=1 .
$$

We may assume, without loss of generality, that $k_{1}, \ldots, k_{i}$ are non-negative and that $k_{i+1}, \ldots, k_{n}$ are negative. Put

$$
t=t_{1}^{k_{1}} \cdots t_{i}^{k_{i}} \text { and } t^{\prime}=t_{i+1}^{-k_{i+1}} \cdots t_{n}^{-k_{n}} .
$$

Notice that $q_{1}, \ldots, q_{2 n}, \pi_{1}, \ldots, \pi_{n}$ are multiplicatively independent and that (3.7) holds. Further by (1.4) and (3.10), $|t| \geq 3$ provided that $H$ exceeds $C_{2}$. Therefore, by Lemma 2.2, there is a positive number $c^{\prime}$, which is effectively computable in terms of $n$, such that

$$
\left|t-t^{\prime}\right|>|t| /(\log |t|)^{c^{\prime}}
$$


Plainly we may suppose that

$$
c^{\prime} \geq 1
$$

We may assume that

$$
\left|u_{j}-t_{j}\right|<\left|t_{j}\right| /\left(\log \left|t_{j}\right|\right)^{c^{\prime} 9(n-1) \varepsilon^{-1}}
$$

for $j=1, \ldots, n$ since if there is no $\mathbf{u}$ in $\mathcal{M}_{n}(\mathbb{C})$ with coordinates algebraic numbers of height at most $H$ and degree at most $d$ for which (3.16) holds then the result follows. Define $\theta_{j}$ by

$$
u_{j}=t_{j}\left(1+\theta_{j}\right)
$$

for $j=1, \ldots, n$. Then, by (3.16),

$$
\left|\theta_{j}\right| \leq \frac{1}{\left(\log \left|t_{j}\right|\right)^{c^{\prime} 9(n-1) \varepsilon^{-1}}}
$$

for $j=1, \ldots, n$. Now

$$
\left|z_{j}-t_{j}\right|<\left|z_{j}\right| /\left(\log \left|z_{j}\right|\right)^{c}
$$

for $j=1, \ldots, n$ and so for $H>C_{3}$, by (1.4),

$$
\left|t_{j}\right| \geq\left|z_{j}\right| / 2 \geq\left|z_{j}\right|^{1 / 2}
$$

for $j=1, \ldots, n$. By (1.4), (3.18) and (3.19)

$$
\left|\theta_{j}\right| \leq\left(\frac{2}{(\log H)^{\varepsilon}}\right)^{c^{\prime} 9(n-1) \varepsilon^{-1}}
$$

hence, for $H>C_{4}$,

$$
\left|\theta_{j}\right| \leq \frac{1}{(\log H)^{c^{\prime} 8(n-1)}}
$$

for $j=1, \ldots, n$. By (3.17) and (3.12)

$$
1=t_{1}^{k_{1}} \cdots t_{n}^{k_{n}} \prod_{j=1}^{n}\left(1+\theta_{j}\right)^{k_{j}}
$$

and so

$$
t_{i+1}^{-k_{i+1}} \cdots t_{n}^{-k_{n}}=t_{1}^{k_{1}} \cdots t_{i}^{k_{i}} \prod_{j=1}^{n}\left(1+\theta_{j}\right)^{k_{j}}
$$

By (3.11) and (3.20)

$$
\left(1-\frac{1}{(\log H)^{8 c^{\prime}(n-1)}}\right)^{n C_{1}(\log H)^{n-1}} \leq\left|\prod_{j=1}^{n}\left(1+\theta_{j}\right)^{k_{j}}\right| \leq\left(1+\frac{1}{(\log H)^{7 c^{\prime}(n-1)}}\right)^{n C_{1}(\log H)^{n-1}}
$$

and so, for $H>C_{5}$,

$$
1-\frac{1}{(\log H)^{5 c^{\prime}(n-1)}} \leq\left|\prod_{j=1}^{n}\left(1+\theta_{j}\right)^{k_{j}}\right| \leq 1+\frac{1}{(\log H)^{5 c^{\prime}(n-1)}} .
$$

By (3.13), (3.21) and (3.22)

$$
\left|t-t^{\prime}\right| \leq \frac{|t|}{(\log H)^{5 c^{\prime}(n-1)}}
$$


Notice that by (1.4), (3.11) and (3.10)

$$
|t| \leq(2 H)^{n C_{1}(\log H)^{n-1}}
$$

so

$$
\log |t| \leq C_{6}(\log H)^{n} \leq C_{6}(\log H)^{2(n-1)} .
$$

Thus, for $H>C_{7}$,

$$
\log |t| \leq(\log H)^{(5 / 2)(n-1)} .
$$

It follows from (3.23) and (3.24) that

$$
\left|t-t^{\prime}\right| \leq|t| /(\log |t|)^{2 c^{\prime}}
$$

Now from (3.14) and (3.25) we see that

$$
2 c^{\prime}<c^{\prime}
$$

and this is false. Our result now follows.

\section{References}

[LoxP83] J. H. Loxton and A. J. van der Poorten, Multiplicative dependence in number fields, Acta Arith. 42 (1983), $291-302$.

[PSSS18] F. Pappalardi, M. Sha, I. E. Shparlinski and C. L. Stewart, On multiplicatively dependent vectors of algebraic numbers, Trans. Amer. Math. Soc. 370 (2018), 6221-6244.

[PLox77] A. J. van der Poorten and J. H. Loxton, Multiplicative relations in number fields, Bull. Austral. Math. Soc. 16 (1977), $83-98$.

[SSS] M. Sha, I. E. Shparlinski and C. L. Stewart, On the distribution of multiplicatively dependent vectors, to appear.

[Ste18] C. L. Stewart, Sets generated by finite sets of algebraic numbers, Acta Arith. 183 (2018), 193-200.

[Tij73] R. Tijdeman, On integers with many small prime factors, Compos. Math. 26 (1973), 319-330.

[Tij74] R. Tijdeman, On the maximal distance between integers composed of small primes, Compos. Math. 28 (1974), 159162.

\section{C.L. Stewart}

Department of Pure Mathematics

University of Waterloo

Waterloo, Ontario, Canada

N2L 3G1

e-mail: cstewart@uwaterloo.ca 